\title{
Civilisations
}

Revue internationale d'anthropologie et de sciences

humaines

56 | 2007

Après la catastrophe

\section{Comme une écaille sur le mur : à propos de «Le Dernier caravansérail (Odyssées) », un spectacle en deux parties du Théâtre du Soleil}

\section{Françoise Lauwaert}

\section{OpenEdition}

\section{Journals}

Édition électronique

URL : http://journals.openedition.org/civilisations/568

DOI : $10.4000 /$ civilisations.568

ISSN : 2032-0442

\section{Éditeur}

Institut de sociologie de l'Université Libre de Bruxelles

\section{Édition imprimée}

Date de publication : 1 décembre 2007

Pagination : 159-182

ISBN : 2-87263-016-3

ISSN : 0009-8140

\section{Référence électronique}

Françoise Lauwaert, « Comme une écaille sur le mur : à propos de « Le Dernier caravansérail (Odyssées) », un spectacle en deux parties du Théâtre du Soleil », Civilisations [En ligne], 56 | 2007, mis en ligne le 01 décembre 2010, consulté le 01 mai 2019. URL : http://journals.openedition.org/civilisations/568 ; DOI : 10.4000/civilisations.568 


\title{
Comme une écaille sur le mur A propos de "Le Dernier caravansérail (Odyssées)", un spectacle en deux parties du Théâtre du Soleil
}

\author{
Françoise LAUWAERT
}

Résumé : Depuis sa fondation il y a maintenant quarante ans, le Théâtre du Soleil travaille sur l'histoire et met en scène les déchirements de notre époque. Son dernier spectacle, Le Dernier Caravansérail, a pour point de départ la rencontre des réfugiés du centre de Sangatte (France: Pas de Calais). Ce spectacle, nourri de récits de vie collectés en des lieux divers, prête à la comparaison avec le travail de l'anthropologue. Les thèmes que nous avons souhaité aborder ici sont : le recours à la langue originale, la production d'un récit, la confrontation à l'image et au cliché, la représentation, la transmission d'une expérience, la reconstruction des vies après la catastrophe et à travers l'exil et le déracinement.

Mots-clés : exil, récit, langue originale, théâtre.

Summary: Since its foundation 40 years ago, the Théatre du Soleil has used History as subject matter, and performs material related to the rifts of our time. Its latest show, Le Dernier Caravansérail, starts with an encounter between refugees from the Sangatte centre (France: Pas de Calais). This show, full of life narratives collected in diverse locations, can be compared with the work of the anthropologist.

The themes developed in this paper are the recourse to original languages, the production of a narrative, the debunking of false images and stereotypes, the representation, transmission of an experience, the reconstruction of lives after a disaster, and through exile and uprooting.

Key words: exile, narrative, original language, theater. 
C'était mon premier voyage, et c'était un long voyage. Tout était nouveau à mes yeux, et quelque peu terrifiant. Car j'étais seul, j'étais un enfant. Je n'avais en ma compagnie aucun membre de ma famille, je n'avais aucun ami qui me donne du cour. Tout était halètement Récit de Mansour, réfugié afghan

Ce sont des choses en suspens, comme une paupière ouverte sur un monde. On aperçoit des choses et ça se referme et on comprendra la suite, peut-être dans un autre pays. ... C'est un spectacle fait de battements de paupières et tout d'un coup d'hallucinations où les yeux restent écarquillés.

Ariane Mnouchkine, lors de la répétition du 5 novembre 2002

Peut-on être étranger et heureux? L'étranger suscite une idée neuve du bonheur. Entre fugue et origine : une limite fragile, une homéostase provisoire. Posé, présent, parfois certain, ce bonheur se sait pourtant en transit, comme un feu qui ne brille que parce qu'il consume. Le bonheur étrange de l'étranger est de maintenir cette éternité en fuite ou ce transitoire perpétuel. (Kristeva, $1988: 13$ )

Chère Nadereh, Nadereh dja... Les paroles s'impriment sur la soie du rideau, tandis que s'élève la voix d'Ariane lisant la lettre (datée du 2 avril 2003) qu'elle adresse à une réfugiée afghane rencontrée en Indonésie. Avec un léger décalage vient la traduction en persan, cette langue qui fut parlée par les caravaniers sur toute la longueur de la Route de la Soie, avant que les intégristes ne martèlent pour la première fois les visages des bouddhas des oasis. Le silence se densifie, le prologue prend le temps de se dire sur la belle musique de Jean-Jacques Lemêtre. Nous sommes invités au voyage par la séduction de cette langue qui nous est pleinement donnée à écouter.

Le Dernier caravansérail (Odyssées) est un spectacle en langue originale qui parle de l'exil, de la guerre et de la perte, de l'hospitalité et de la non-hospitalité. Ses principaux protagonistes sont « ceux que l'on nomme réfugiés, clandestins, migrants, et qui, entre eux, s'appellent plus noblement les voyageurs ${ }^{1} »$. Il comporte deux parties, Le Fleuve cruel et Origines et destins, et se présente sous la forme d'une succession de scènes ${ }^{2}$ se déroulant en plusieurs points du monde : les pays que l'on quitte et auxquels il faut parfois revenir (Iran, Afghanistan, Russie, Irak...), les lieux où l'on s'échoue (Villawood, quelque part en Australie) et les lieux où l'on attend : Sangatte, cet immense centre d'hébergement mis en place par la Croix-Rouge en septembre 1999, où étaient rassemblés les réfugiés en attente et en espoir de passer en Angleterre :

Pendant un moment, on a pu entrer à Sangatte comme visiteur. C'est en cela que les gens qui appelaient Sangatte un camp exagéraient un tout petit peư ${ }^{3}$. C'était un

1 Note d'intention d'Ariane Mnouchkine pour la réalisation du film basé sur le spectacle, tourné en mai et juin 2005 à la Cartoucherie.

2 Dix-neuf pour la première et vingt-trois pour la deuxième.

3 On se reportera à ce propos à l'article de Denis Peschanski dans le catalogue de l'exposition de photos que Jacqueline Salomon (2002) a consacré à ce lieu. 
centre d'hébergement d'urgence ouvert. [... ] C'est cela aussi qui a extrêmement touché Ariane: Sangatte, c'était le dernier caravansérail, c'était la dernière halte, un endroit où les humanités pouvaient se rencontrer. Bien sûr, il y avait des voyous, mais aussi des poètes, des femmes, des marchands... C'était un îlot où les gens soufflaient avant de repartir dans leur voyage périlleux. C'est devenu de moins en moins cela après, parce que les conditions d'accueil étaient trop mauvaises, il y avait plus de huit cents personnes qui passaient par semaine. C'est devenu un enfer. Mais au début, c'était autre chose $e^{4}$ (Charles-Henri).

Le Fleuve cruel a d'abord été donné comme un spectacle indépendant. J'étais allée le voir à Paris au printemps 2003, comme je vais voir tous les spectacles du Théâtre du Soleil depuis plus de vingt ans, et c'est alors que s'est faite la rencontre. Je venais de soumettre au comité de rédaction de la revue Civilisations un projet de numéro sur le thème « Après la catastrophe », et j'ai perçu une grande proximité entre ce spectacle et la vision que j'avais alors de ce pourrait être ce numéro. Il me semblait voir dans ce travail théâtral une démarche proche de celle de l'anthropologue, qui tente de dire quelque chose du monde d'aujourd'hui sans trop trahir. C'est sur la base de cette intuition qu'avec l'aide précieuse d'Isabelle Henrion-Dourcy, j'ai pu rencontrer Charles-Henri Bradier, l'assistant d'Ariane Mnouchkine. La réponse a été au-delà de mes espérances : j'étais invitée aux répétitions de la deuxième partie du spectacle qui serait montrée fin novembre, je pourrais rôder sur les lieux, consulter les journaux des répétitions, faire des entretiens avec les comédiens, partager leurs repas et un peu de leur temps. Ce travail s'est poursuivi pendant près de deux ans.

Ton travail, c'était comme si nous allions être les Indiens d'Amazonie et que tu viennes nous " étudier », etça m'intéressait beaucoup d'être les Indiens d'Amazonie. Mais au fond, ça n'a pas été tout à fait ça. Je me disais que c'est bien d'être des Indiens d'Amazonie, parce que c'est vrai d'une certaine façon pour les gens du Théâtre du Soleil. On est comme une sorte de vestige et peut-être en même temps à l'avant-garde. Les Indiens d'Amazonie sont à la fois un vestige et à l'avant-garde d'une certaine attitude écologique. Et donc, je trouvais ça drôle qu'une ethnologue vienne et nous étudie comme une ethnie étrange (Ariane).

En effet, ce n'était pas les Indiens d'Amazonie, bien qu'il y ait quelque chose de la tribu dans une troupe comme celle du Théâtre du Soleil. Plus que le déchiffrement des rituels de cette " ethnie étrange », ce qui m'intéressait, c'était le travail qu'elle avait accompli pour ce spectacle particulier : la mise en récit et la mise en images, la traduction, la transmission, le passage. Il ne sera donc pas question ici d'analyser la performance théâtrale en tant que telle, ni de replacer ce spectacle dans l'histoire déjà longue du Théâtre du Soleil et de ses engagements, ni de porter un jugement sur la pertinence politique de la démarche des auteurs de ce travail collectif, mais bien de répertorier les étapes de la construction de ce Caravansérail.

4 Les citations sont extraites d'entretiens avec Ariane Mnouchkine, Charles-Henri Bradier et plusieurs comédiens, seuls ou en groupe, réalisés entre l'automne 2003 et le printemps 2005 : Shaghayegh Behesti (dite Shasha), Duccio Bellugi-Vanuccini, Virginie Colemyn, Delphine Cottu, Maurice Durozier, Sarkaw Gorany, Astrid Grant, Elena Loukiantchikova-Sel, Jean-Charles Maricot et Serge Nicolaï. On trouvera aussi quelques extraits des interventions d'Ariane Mnouchkine lors des répétitions. Tous se sont prêtés à l'exercice avec la plus grande gentillesse, mettant en pratique les traditions d'hospitalité du Théâtre du Soleil. Ma reconnaissance à leur égard est à la mesure de cet accueil chaleureux. 


\section{Ceci n'est pas un jour comme un autre jour}

Fondé il y a quarante ans et installé depuis 1970 dans la Cartoucherie de Vincennes ${ }^{5}$, le Théâtre du Soleil accueille son public dans ses beaux bâtiments du $19^{\mathrm{e}}$ siècle selon un rituel (presque) immuable : bien à l'avance (les places ne sont pas numérotées), les spectateurs entrent dans une première nef qui sert de vestibule à la salle de spectacle. Là, ils peuvent consulter ou acheter les livres qui ont inspiré la création ${ }^{6}$, discuter entre eux et admirer l'impressionnante collection d'instruments de musique de Jean-Jacques Lemêtre. Dans la salle, sous les gradins, sont installées les loges, qui sont à la fois séparées et visibles, afin que les spectateurs qui le désirent puissent entrevoir quelque chose du processus de métamorphose. Sur les murs, le grand planisphère où figurent les lieux de provenance des voyageurs s'ajoute aux traces laissées par les spectacles précédents : une fresque d'inspiration tibétaine, une miniature et des calligraphie persanes... près de la porte, un quatrain de Li Bai, grand poète chinois du $8^{\mathrm{e}}$ siècle.

Le théâtre ouvre une heure précise avant le début du spectacle. Ce moment est entouré d'une certaine solennité : de l'autre côté de la double porte, dans un silence complet, Ariane rappelle aux comédiens que les spectateurs sont attendus. Ce petit rituel est redoublé par un autre accompli quelques minutes avant l'ouverture du rideau par les comédiens, discrètement, à l'abri des écrans de tulle qui masquent en partie les musiciens.

Cette petite réunion, elle est nécessaire pour qu'on se rappelle que "ceci n'est pas un jour comme un autre jour $»^{7}$. Au théâtre, c'est chaque jour qui n'est pas un jour comme un autre. Parce que les gens qui sont là sont venus célébrer quelque chose, et c'est chaque jour différent. Très souvent, on se rappelle que pour plein de gens, c'est la première fois qu'ils viennent au théâtre et il y en a toujours aussi pour qui c'est la dernière fois. Quand tu penses ça, tu sais bien que tu vas faire un acte qui n'est pas banal. Ce n'est pas banal de se charger de l'émotion, de la réflexion, du silence, de la vulnérabilité des gens pendant trois heures, cinq heures. Mais il suffit là-dedans d'une réflexion vulgaire pour tout casser. Tout ce rituel, les comédiens qui deviennent les personnages, qui me disent bonjour en anglais... il suffit d'un hargneux qui ne veut pas rentrer dans ce monde un peu magique, poétique, pour qu'on retombe sur le plancher des vaches. Le problème, c'est que la scène, ce n'est pas le plancher des vaches (Ariane).

Dans la première salle, des repas sont servis par les comédiens. Cen'est pas anecdotique, la référence à la nourriture est constante dans la troupe. Elle est à prendre au sens propre -

5 Cette histoire est racontée par Guy-Claude François à Béatrice Picon-Vallin lors d'un entretien réalisé au Théâtre du Soleil le 5 février 2004 et diffusé sur le site http://www.lebacausoleil.com. D'autres troupes s'installeront peu de temps après à la Cartoucherie : le Théâtre de la Tempête, l'Epée de Bois et l'Atelier du Chaudron en 1972, le Théâtre de l'Aquarium, en 1973. Pour plus de renseignements sur ce lieu, on se référera à l'article de Joël Cramesnil, «L'historique de la Cartoucherie », également diffusé sur le site et à son livre dernièrement paru (Cramesnil 2004).

6 «Mnouchkine rêve non seulement d'acteurs, mais de spectateurs instruits et elle facilite l'accès immédiat aux sources ». Cet aspect, souligné par Georges Banu (2002) se retrouve dans le soin, il faudrait dire l'amour, avec lequel est construit le site Internet destiné en premier lieu aux lycéens préparant le bac Théâtre. Ce site a été pour moi une mine inépuisable.

7 Ariane Mnouchkine fait référence au rituel du Seder, dont nous avons longuement parlé lors d'un entretien qui n'a été repris que très partiellement ici. 
on est bien nourri au Théâtre du Soleil : spectateurs, comédiens et visiteurs -, mais aussi, et peut-être surtout, comme métaphore :

Euripide dit, en parlant des auteurs: "nous nous asseyons tous au banquet homérique. " Nous mangeons tous Homère et heureusement qu'il y a des grandes gens comme cela à manger. On mange tous Homère, Shakespeare... et des visions, des récits, des chroniqueurs. Un artiste, un artisan, un comédien, il se nourrit. L'inspiration ne vient pas du vide (Ariane).

L'hospitalité, la convivialité sont des thèmes très présents au Théâtre du Soleil qui, rappelons-nous, hébergea trois cents et quelque sans-papiers en $1995^{8}$. Ce n'est donc pas un hasard si une violente dispute éclate avec les organisateurs d'un festival à propos de l'accueil qu'ils entendent réserver à une troupe balinaise venue représenter un Topèng à la Cartoucherie. Ariane veut qu'ils restent à dîner après le spectacle, avec les comédiens ; les organisateurs souhaitent qu'ils prennent le bus le plus rapidement possible pour rejoindre leur hôtel. «Je ne suis pas obligée de les recevoir aussi mal que vous les auriez reçus si ce n'avait pas été ici» rétorque Ariane, qui interdit aux organisateurs de mettre les pieds dans son théâtre.

Dans ce lieu travaillent des comédiens, des costumiers, des éclairagistes, des informaticiens, graphistes, vidéastes..., tous ceux qui se chargent des tâches administratives et de l'accueil et, bien sûr, des musiciens. Tous contribuent à l'entretien des locaux et travaillent aux cuisines, un lieu qui est, comme souvent, le cœur vivant où s'opèrent les rencontres et les échanges. Tous les comédiens, et bien d'autres encore, participent à l'élaboration des décors et à la collecte de la documentation. Les salaires sont les mêmes pour tous. L'ambiance est à la fois libre et disciplinée. Des petits papiers sont collés partout : " J'insiste gentiment, mais fermement pour que vous fermiez bien les robinets »; "Le chat n'a vraiment rien à faire dans les bureaux »; "Chers amis, ces cendriers sont là précisément pour que vous ne jetiez pas les mégots partout...». Cela prête à sourire, mais plus de quatre-vingts personnes travaillent en ces lieux et quiconque a vécu ne serait-ce que quelques semaines en communauté comprend aisément la raison de ces rappels à l'ordre. Cette entreprise, qui peut paraître utopique, vit, crée et travaille intensément depuis quarante ans. C'est une réussite fragile et menacée; les menaces provenant moins sans doute des dissensions internes, ruptures et autres départs (crises qui font partie de l'histoire de la troupe et qu'elle arrive tant bien que mal à digérer), que de l'incertaine évolution de notre planète :

Le 11 septembre a été pour tout le monde, et pour Ariane, un choc absolu, qui l'a remise en cause profondément. Et très vite après, en stage, elle a parlé de l'urgence : urgence du théâtre, théâtre de l'urgence. C'est quelque chose qui est venu aussi de sa perception des menaces qui pèsent sur un mode de fonctionnement comme le nôtre. Sur un groupe humain comme le nôtre, une troupe qui est encore subventionnée et qui réussit pendant huit mois à travailler sur le monde contemporain sans avoir le souci ni la pression du résultat. Et ce laboratoire, c'est quelque chose qu'on sent de plus en plus menacé (Charles-Henri).

8 Ces événements sont la source directe du spectacle Et soudain des nuits d'éveil, donné à la Cartoucherie en 1997. 
Pratiquement chaque jour viennent des visiteurs : anciens comédiens (quelques-uns, pas tous, les ruptures ne sont pas toujours veloutées), écrivains, témoins, journalistes (accueillis, mais avec une certaine réticence), musiciens, historiens ou philosophes. Cette " maison du présent ${ }^{9}$ » accueille des troupes étrangères, organise des événements (colloque sur le Tibet, débat sur la Constitution européenne...), reçoit, parfois pour de longs mois, l'enseignement d'un maître de tambours coréens, d'un acteur de Noh, d'une danseuse du Tibetan Institute of Performing Arts. Au moment où cet article est écrit (fin 2005), les comédiens sont au nombre de trente-huit. Certains sont là depuis de longues années et ont participé à de nombreux spectacles, d'autres sont partis puis revenus, d'autres enfin viennent d'entrer dans la troupe après un stage ou dans le sillage d'une tournée en Australie, au Japon ou en d'autres lieux. Dans ce spectacle-ci, Sarkaw Gorany, un comédien d'origine kurde, qu'Ariane avait rencontré à Sangatte, incarne plusieurs personnages importants :

\begin{abstract}
Jean-Pierre $A^{* * *}$, du GISTI (Groupe d'information et de soutien aux travailleurs immigrés) allait à Sangatte régulièrement, puisque c'est un de leurs terrains de travail, d'investigation, de revendication. Et il avait dit à Ariane : " là-bas, il y a un garçon, un Kurde, qui s'appelle Sarkaw Gorany. C'est un acteur qui a beaucoup entendu parler de toi, et il voudrait te rencontrer. Il est venu en France parce qu'il voulait faire du théâtre et parce qu'il aime la France. C'est un fou de littérature, un fou de Rimbaud. Lui, il ne veut pas aller en Angleterre, il a déjà ses papiers en France puisqu'il parle très bien français ». Il traduisait à cette époque-là pour les médiateurs, pour la gendarmerie quelques fois, pour les gens qui pouvaient rencontrer les réfugiés. Et Ariane a dit à Jean-Pierre : "Ça tombe bien, parce que je voulais aller à Sangatte ». Et elle est allée à Sangatte rencontrer Sarkaw et les premiers tours qu'elle a faits à Sangatte, c'était avec lui (Charles-Henri).
\end{abstract}

Pendant les répétitions, ce vaste espace est occupé par les décors : l'atelier de fabrication, puis le vestibule et la salle proprement dite, où sont installés les décors prêts, l'immense collection de vêtements dans lesquels puisent les comédiens, et la documentation (livres, photos, articles de journaux, disposés sur de grandes tables). Près des coulisses sont rangés de gros classeurs contenant le journal des répétitions : un par semaine, où figurent le synopsis des improvisations, le relevé des interventions d'Ariane et des comédiens, et de nombreuses photos. Les répétitions sont également filmées en vidéo, et souvent les comédiens se réfèrent à ces archives, qui sont immédiatement disponibles. Tout est très bien rangé au Théâtre du Soleil. Cette volonté d'ordre, cette horreur du brouillon, de la perte de temps, de l'anarchie participe d'un projet éthique et esthétique englobant la vie de la troupe comme la construction des spectacles : « Mnouchkine développe une esthétique de l'équilibre qui écarte les menaces de désordre et se réclame d'un ordre architectural. Une passion ordonnée. Une perspective strictement cartographiée » (Banu 2002).

\title{
Retrouver le fragment étincelant
}

«Il faut éviter d'être illustratif, allégorique, symbolique. Il faut que ce soit comme un fragment de vie, comme une écaille sur le mur... » (Ariane, répétition du 2 octobre 2002). Le Dernier caravansérail est construit comme une succession de scènes se déroulant

9 Hélène Cixous (2000: 10). 
en des temps et des lieux différents. Les comédiens n'illustrent pas des récits ou des témoignages; ils sont appelés à imaginer des situations et des dialogues à partir de leurs affinités avec les destins des voyageurs, ceux que Charles-Henri Bradier définit comme " ces autres acteurs - ceux qui ne sont pas sur le plateau, mais qui sont aussi des acteurs du Dernier caravansérail »:

Il y a beaucoup de spectateurs qui demandaient : " avec combien de réfugiés vous travaillez? Vous les avez trouvés où ? Comment ça se passe de vivre avec eux? »etc. et c'était quelquefois très difficile de leur faire comprendre que tous ceux qui jouent dans ce spectacle sont des acteurs professionnels, que même les acteurs qui viennent de loin, comme Shasha, Sarkaw ou Elena, étaient des acteurs avant d'arriver au Théâtre du Soleil. Il y a un travail de " composition » - ce n'est pas un terme qu'on utilise au Théâtre du Soleil, on dirait plutôt un travail d'incarnation - qui a été mené par les acteurs lors des improvisations. Et ce sont les improvisations qui ont nourri petit à petit la succession des tableaux. L'improvisation a d'ailleurs nourri la conception générale du spectacle, c'est-à-dire, à la fois l'écriture dramatique et l'écriture scénographique. Ce sont les comédiens eux-mêmes qui, en travaillant sur chaque espace, chaque situation, créaient leur propre espace de jeu. Si Ariane retrouvait l'émotion qu'elle avait pu ressentir en écoutant les vrais réfugiés, elle savait qu'on était sur le bon chemin pour faire venir une scène. C'est comme cela qu'on a réussi, par couches successives, à passer de la réalité de l'émotion vécue à la transposition au théâtre. Après, Ariane est venue préciser à certains moments des choses auprès des comédiens. Elle leur disait : " mais en Iran, on ne peut pas tout à fait dire cela comme ça... » Donc après, le travail documentaire a été très important de la part des comédiens et d'Ariane (Charles-Henri).

Chaque matin, les comédiens se réunissent en cercle sur le plateau et font des propositions : "Un atelier de couture en Angleterre : le patron distribue le travail à ses ouvrières, qui sont des réfugiées. Soudain, une dispute éclate entre deux d'entre elles, une Russe et une Tchétchène... " Des petits groupes se constituent, les comédiens discutent entre eux et élaborent un canevas. Au cours de ce « concoctage », le trait s'affine et très vite on passe au choix des costumes, au maquillage et au décor. Des improvisations, parfois très abouties, il y en a eu plus de trois cents. Ce travail n'a pu être possible que par la mise en place d'un type très particulier de décor. J'y reviendrai plus longuement. Notons simplement ici que les lieux multiples où se déroulent les fragments d'histoires qui constituent la substance même du spectacle sont figurés par des chariots : des sortes de roulottes poussées sur le plateau par d'autres comédiens laissés disponibles par la scène du moment.

Toutes les répétitions, depuis le début, se font en musique - lorsque Jean-Jacques Lemêtre $^{10}$ doit s'absenter, le travail s'arrête. La musique n'est en rien une illustration sonore, elle emporte les acteurs et les spectateurs «au cœur d'un monde sonore érigé en partenaire » (Banu 2002). Les préparatifs peuvent prendre plusieurs jours. Lorsque les comédiens sont prêts, ils présentent leur improvisation à Ariane et aux autres comédiens.

10 Jean-Jacques Lemêtre, qui se définit comme un musicien de théâtre, participe aux créations du Théâtre du Soleil depuis 1979. Sa présence est essentielle, consubstantielle. Il évoque son travail dans Lemêtre (1998). 


\begin{abstract}
Ariane arrivait en répétitions très tard, vers quatre, cinq heures de l'après-midi, parce que la consigne, c'était [...] de préserver la surprise, de conserver l'apparition. Ariane voulait voir apparaître. Il fallait qu'on découvre ces personnages les uns après les autres, et cette accumulation pouvait donner le monde. La scène devenait un planisphère visité par chaque être vivant de chaque pays blessé (CharlesHenri).
\end{abstract}

Ce moment de la première apparition était souvent le moment du verdict, car de nombreuses propositions n'ont jamais dépassé ce stade. Pour d'autres commençait le long travail d'ajustage et de soumission à deux exigences : celles de dire la vérité, ou du moins une vérité sur ces pays, ces gens, ces situations, et celle de faire un travail théâtral, de construire une métaphore.

Quand on travaille les Australiens, et je suis sûre que c'est pareil pour les Iraniens ou pour les Afghans, on trouve d'abord les clichés. Mais il faut laisser partir l'improvisation, même si ce n'est pas du tout un personnage juste. C'est pour produire l'" essence » en quelque sorte, puis on travaille plus tard pour arriver à quelqu'un qu'on pourrait rencontrer là-bas. Les répétitions ont duré sept mois et il y a peu de personnages qui n'ont pas eu le temps d'être développés. Beaucoup de scènes ont été créées après un certain temps, mais il en reste quelques-unes des débuts aussi. Alors là, on a commencé à apprendre quelque chose qui marchait (Astrid).

Il fallait aussi que la scène puisse être reçue à la fois comme une unité en soi et comme une ouverture vers un immense non-dit. En ce sens, le parallèle avec la scansion en psychanalyse me paraît éclairant, elle qui vient donner dans l'après-coup valeur et sens au fragment arraché au continu.

\title{
Raconter, c'est un mot qui revient souvent chez Ariane
}

Pour décrire plus finement ce processus, peut-être faut-il partir de ce que signifie « raconter» pour Ariane et pour les comédiens du Soleil.

Raconter, c'est un mot qui revient souvent chez Ariane. Depuis les années 1980, pour elle, le théâtre, c'est raconter des histoires. Tout le monde raconte, mais pour elle, c'est quelque chose d'essentiel. Si on est conscient de vouloir " entrer dans l'Histoire ", on n'y entre jamais, dans l'Histoire. Un artiste, quand il est en train de travailler, il ne se donne pas des concepts, il libère son imagination. Ce qu'Ariane fait, souvent, ce n'est pas partir d'un concept ou d'une idée. C'est très "idée » de dire : "Je-raconte-l'histoire-d'une-génération-à-une-autre », c'est un concept qui peut bloquer le travail. On est parti très modestement (Sarkaw).

Ce spectacle est parti de l'intérêt humain, on pourrait presque dire "militant» d'Ariane pour le centre de réfugiés de Sangatte ${ }^{11}$ (plus tard, il y aura les centres de détention d'Indonésie et d'Australie). Lors de chacune de ses visites en ces lieux, elle rencontrait des réfugiés et leur demandait de raconter :

Au début, j'avais beaucoup de mal à leur faire raconter des choses concrètes. Ils m'abreuvaient d'opinions, et je ne peux rien en faire, moi, d'opinions. A un

11 Jean-Charles Maricot rappelle que le travail sur ce spectacle commença peu de temps après le choc du 21 avril 2001, où l'on vit Le Pen arriver en deuxième place du premier tour des élections présidentielles. 
moment, je leur ai dit: "Écoutez, ne me racontez pas ça, parce que je sais, je sais même des choses que vous, vous ne savez pas, et que je peux vous raconter $》$. [...] Donc, je leur ai dit: "Ce n'est pas ça qui m'intéresse. Ce qui m'intéresse, c'est des choses concrètes de ta vie: comment était ta maison quand tu es parti, comment était le camion. Et puis, un jour quand tout sera arrangé, quand tu seras en Angleterre, que tu auras des enfants ou même des petits-enfants, qu'est-ce que tu leur raconteras? Le pire moment ou le meilleur moment... qu'est-ce que tu leur raconteras? "Et c'est comme cela que je suis arrivée petit à petit à obtenir des récits. Et les interviews, j'en suis fière parce que ce sont des récits. Si j'écoute les cassettes, j'ai des heures de blabla, des heures de baratin, de jugements sur l'amour, le monde entier... et ce n'est qu'au bout d'un moment qu'ils commencent vraiment à me raconter : "un soir, dans un camion, j'étais là et il y a un type qui a raconté une blague et il y a cette femme qui s'est fait arrêter sur la route et je ne sais pas ce qu'elle est devenue... "Il fallait beaucoup de temps, et parfois on allait dîner à Calais, et à ce moment-là, petit à petit, ils se mettaient à raconter vraiment (Ariane).

Aux comédiens, Ariane disait de la même manière : "Imaginez que vous allez raconter cela, un événement de votre vie qui vous tient à cour, qui vous a un jour fait trembler, qui vous a fait rire, qui vous a fait pleurer, et vous allez le raconter comme si vous le racontiez à vos petits-enfants, des années après ». Même si elles renvoient à des situations réelles, ces histoires ont quelque chose du rêve ou de l'épopée : c'est ce que (se) racontent les voyageurs dans leurs lieux de confinement. Cette dimension est redoublée par l'insertion de documents sonores (qui portent dans le langage du Théâtre du Soleil, le nom mystérieux d'expériments), où l'on entend des poèmes, des chansons, le terrifiant édit du Mullah Omar ordonnant le bombardement des bouddhas de Bâmyân, la très belle lettre qu'envoie Sarkaw Gorany à son père, lui-même en exil, et les voix multiples de ces voyageurs qui relatent un épisode traumatique ou évoquent les douceurs du pays avant:

Lorsque j'ai passé la porte de la maison de mon père, un tel parfum s'est dégagé ! Les arbres venaient de bourgeonner. Les poiriers, les pêchers...

Ils venaient juste de donner des fleurs!

J'ai passé la porte et mon père s'est écrié : "Viens donc t'asseoir ici!"》

Il me serrait si fort dans ses bras, il disait : "Assieds-toi et mange donc!" "

Puis, quand je rentrais chez mon beau-père, il s'écriait : "Pourquoi es-tu partie?

En ton absence, nous avons perdu le goût du pain tant tu nous as manqué » (récit de Nadereh à la fin de la $12^{\mathrm{e}}$ séquence).

Ces récits, je ne les ai pas donnés au début: je leur faisais écouter après, pour confirmer. Et après, j'ai compris l'impact émotionnel qu'ils avaient sur eux. Sur moi, ils avaient eu un impact très très fort au moment où je les avais écoutés, mais je ne me rendais pas compte qu'ils avaient gardé cet impact même pour quelqu'un qui n'était pas face à la personne. Le premier, je crois que c'était Nadereh que je leur ai fait écouter, et quand je me suis sentie pleurer à nouveau et que j'ai vu tout le monde qui pleurait, je me suis rendu compte qu'il y avait une force dans ce récit. Et c'est là que je me suis dit : on va en entendre aussi, et on a découvert les problèmes de son (Ariane).

La dimension sonore de ce spectacle, soutenue par la musique de Jean-Jacques Lemêtre, est essentielle et elle est renforcée par l'usage que font des comédiens des langues étrangères. Répétons-le : ce spectacle est en VO. Non seulement les extraits de récits des réfugiés sont en langue originale (la traduction apparaissant sur la soie de fond), 
mais les comédiens aussi jouent dans les langues de leurs personnages. Ce qui signifie que les acteurs d'origine russe, kurde, iranienne ou australienne parlent dans leur langue, mais aussi que les autres ont appris les langues de leurs rôles. Ils ont, par exemple, tous pris des cours de persan. Il ne s'agissait pas d'une simple contrainte technique, mais d'une discipline de décentrement. C'est la difficulté de l'étranger « qui doit demander l'hospitalité dans une langue qui par définition n'est pas la sienne «(Derrida $1997: 21$ ) qui était ainsi assumée en miroir par les comédiens ${ }^{12}$.

Pour un acteur français, de passer par la langue étrangère, ça l'oblige à transformer sa voix, à la faire devenir chant. C'est une forme de métaphore aussi, de transposition, qui nous a éloignés du réalisme et qui a beaucoup servi les acteurs, qui avaient besoin de cela pour nourrir leur imaginaire sur ces personnages (Charles-Henri).

Ce passage par les langues étrangères, outre qu'il évoque poétiquement le bruissement de la pluralité des langues de notre modernité, a eu des conséquences très importantes sur la forme même du spectacle. C'est la nécessité du surtitrage, en effet, qui lui a donné sa forme définitive, en mettant fin aux infinies variations des improvisations : "C'est vrai qu'à un moment donné, nous nous sommes rendu compte qu'il y avait un texte à cause de la nécessité de le sous-titrer, autrement, on aurait continué à s'imaginer qu'il n'y avait pas du tout de texte dans ce spectacle » (Ariane). Le recours à la langue originale, enfin, a confronté les acteurs (et les spectateurs) à ces interrogations si présentes dans notre modernité : comment traduire sans trahir ? comment transmettre, «faire passer »?

C'était la première fois que j'étais amenée à devoir traduire avec vraiment une responsabilité. Ce n'était pas une traduction quotidienne, et c'était énorme, parce que les langues sont très différentes. Les mots ou les tournures de phrases sont porteurs de sens ancestraux. Il y a la poésie de la langue, les images qui, si je les traduis mot à mot en français, ne veulent peut-être rien dire, mais qui révèlent des choses et témoignent d'une manière de penser. Alors, je me suis mise à traduire au mot à mot, et au fur à mesure que je traduisais ainsi, je me rendais compte qu'Ariane, à travers l'étrangeté de nos expressions, étrangeté qui pour nous est familière, commençait à percevoir, palper et comprendre cet univers. Et cela m'a soulagée d'un énorme poids (Shasha).

Ce qui nous est donné à voir et à entendre n'est donc pas un collage de récits bruts, proches ou censés l'être d'une vérité documentaire, mais un feuilletage de récits appartenant à différents registres, modulés dans des langues diverses, et subtilement déformés par le double prisme de l'imaginaire et de la mémoire.

\section{Etant comédiens, vous avez déjà eu mille vies}

Par de nombreux ajustements, grâce aux lectures, aux films et aux rencontres, en puisant dans l'expérience des comédiens venus des contrées évoquées et, surtout, en

12 Dans une scène merveilleusement drôle, une infirmière de Sangatte jouée par Delphine Cottu s'adresse à un réfugié iranien dans un savoureux mélange de français et d'anglais : «You have ampoules, Parviz, it is very dangerous, you can have fièvre... and you can mourir, Parviz ». Plaisanterie sur la proverbiale méfiance des Français vis-à-vis de la langue anglaise, méfiance dont n'est d'ailleurs pas dépourvue la comédienne, mais surtout métaphore des limites de la langue maternelle, de cette impuissance qui nous prend quand nous nous heurtons durement aux murs de la langue. Métaphore aussi de la générosité des bénévoles de Sangatte auxquels ce spectacle rend hommage. 
écoutant les récits des réfugiés que tous les participants se sont mis progressivement à récolter, s'est opérée la rencontre avec les personnages.

Chaque personnage est une histoire singulière. Quand on est acteur, il suffit de rendre son corps disponible. Je dis « il suffit», mais c'est un cheminement, tout un travail : avoir le vide nécessaire à l'intérieur et offrir cette enveloppe à une autre âme qui vient à un moment donné et qui est le personnage. Comment ça se passe? Ça dépend des cas, ça dépend des fois. Parfois il y a des personnages qui viennent plus progressivement, parfois il y a des personnages qui viennent d'un coup, par surprise, je dirais.

Dans le cas de Timour, c'est assez étonnant ce qui s'est passé. J'avais été à Sangatte pour accompagner Ariane et il y avait un réfugié kurde qui m'a en quelque sorte nargué toute la journée. C'était déjà un personnage extraordinaire, un homme très costaud, très fort, qui passait son temps à aller aux douches (je me souviens qu'il portait des tongs). Il m'a nargué et moi, je ne lui demandais rien, parce que quand on fait la démarche d'aller comme ça vers les gens pour chercher l'inspiration, il faut être très délicat. Tout au long de cette journée que j'ai passée à Sangatte, j'ai rencontré plusieurs réfugiés. Il y a d'autres personnages que j'interprète dans le spectacle qui ont pris naissance dans cette journée, mais de tous ces réfugiés, c'est lui qui m'a tendu cette sorte de défi: "Tu ne m'auras pas, toi!", mais finalement, c'est ce qu'il désirait, parce que son attitude n'était pas du tout rationnelle, c'était extrêmement provocateur. Donc, je le suivais dans Sangatte, puis il allait dans le fond de ce qui était son coin et il régnait là, en chef de quartier. Ce personnage de Timour, c'est une invention, mais j'avais une image et aussi un challenge. Il y a une alchimie qui s'est produite. Quand ce personnage est arrivé, immédiatement il a existé tel quel et je n'y ai rien changé, ni au costume, ni aux tongs, ni au ventre qu'il avait. C'était ça.

Quand je fais ce personnage de Timour, je me sens kurde, vraiment. Dans la première partie, il y a un moment où je me mets en Timour et je reste ainsi pendant une heure, et quand je rencontre Sarkaw et qu'on a des discussions, je lui demande de raconter, et là, à ces moments-là il me raconte son pays, il me raconte le Kurdistan. C'est un besoin pour moi. C'est un moment qui n'a peut-être rien à voir avec le spectacle, mais en tout cas, je pense que c'est une forme de communication, une forme de connaissance que je ne pourrais pas avoir par moi-même si je n'étais pas à ce moment-là Timour. Pour moi, quand on parle d'incarnation, parfois c'est comme ça. Voilà, je suis Timour (Maurice).

Le travail de dessin que requiert une telle approche est loin d'exclure la complexité. Celle-ci passe notamment par l'entrelacement des temps des récits. Elle passe aussi par une vigilance : la scène ne peut être peuplée de figurants. Quelles que soient la longueur et la fréquence de leurs apparitions, la centaine de personnages incarnés par les acteurs sont tous pourvus d'un nom, d'une généalogie, d'une famille, d'amis et ennemis ${ }^{13}$, d'une

13 Les improvisations couvraient un champ bien plus vaste que le spectacle abouti. Citons au hasard : des réfugiés nord-coréens perdus dans leurs froides forêts, un radeau chargé de Papous !!! (commentaire d'une comédienne : "ça, ce sera pour le bonus, il y avait trop de maquillage! »), une salle de boxe en Afghanistan, un parc en Iran... Il y avait des djinns, des cosmonautes, des travestis, un Père Noël. De cet extraordinaire luxe d'imaginaire, les spectateurs n'ont vu que la trace : « Nous avons improvisé des scènes avec des djinns, parce que les djinns, ça fait partie de ce monde-là ... Finalement, il n'est rien resté de ces scènes plus légendaires, plus mythologiques qu'on a faites. Mais par contre, dans mon esprit, parfois, je sens que les djinns sont là » (Maurice). 
histoire nourrie par un énorme travail invisible. "Il nous faut de ces exactitudes fertiles, qui vous font revivre des choses déjà vécues, dans une vie antérieure, parce qu'étant comédiens, vous avez déjà eu mille vies », dit Ariane Mnouchkine lors de la répétition du 23 octobre 2002. Ces mille vies, nous en percevons bien sûr des éclats lors du spectacle et elles ont laissé leurs traces dans les personnages, qui venaient accompagnés d'une constellation de mondes possibles :
Moi, toutes les trois cents et quelque improvisations sont dans mon cour. Très souvent entre nous, on a un petit rituel, on essaye de se remémorer, de sortir des tiroirs de l'oubli une improvisation et elles nous reviennent en pleine mémoire. Ça nous aide beaucoup pour le spectacle, parce que c'est une toute petite partie qui est là, et pour nous, c'est mille et une nuits, et les Mille et une nuits correspondent avec le présent. Chaque scène qu'on voit correspond à quelque chose d'invisible qui pour nous est derrière et qui a un lien direct. Chaque personnage a été dans quinze épopées. On en voit une, il y a les quatorze autres (Shasha).

Certains acteurs sont restés dans un registre relativement proche, en jouant par exemple dans leur langue. D'autres, au contraire, sont allés chercher leurs personnages au plus loin. Elena, actrice russe originaire de Moscou, incarne Claudia, une femme échouée à Sangatte, qui consent, puis collabore à la prostitution de sa fille. Elle franchit ainsi la ligne invisible et essentielle qui sépare les vilenies admises de celles qui sont vraiment réprouvées dans ce lieu où tant de choses licites et illicites se passent. Le caractère irréversible de sa déchéance lui est sans cesse rappelé : par sa fille, par les passeurs, qui refusent de lui accorder le passage, par son père, enfin, lorsqu'elle revient en Russie, après avoir échoué dans sa recherche de «la belle Europe ». Devenue un déchet, ce personnage presque beckettien s'anéantit :

Ce qu'elle va devenir, Claudia? C'est le printemps, mais quand je sors, il y a l'espace nu ... une grande étendue de neige. C'est sûr qu'elle finit mal. En Russie, c'est très dur quand la famille vous rejette. Quand j'ai fait cette création, je n'ai pas joué, j'existais, c'est tout, et je ne sais pas comment. C'est mystérieux parce que je ne sais pas comment j'ai trouvé ce personnage, comment j'ai trouvé l'histoire (Elena).

Serge Nicolaï, en revanche, éprouve davantage le besoin de se masquer par la langue, le costume et un maquillage très appuyé, de se confronter au repoussant :
Eclats de guerre (Boulat 2002), sur la Bosnie, est un des livres qui m'a le plus repoussé. Les gars dans ce bouquin, ce sont des jeunes avec le chapeau haut-de- forme et tout... tu sens que tout a été pillé. Ils sont habillés avec des vêtements de pillage, il y a la tenue militaire et le manteau de fourrure. Cette cruauté-là, cette violence-là, cette froideur-là, cette odeur de viande, la marchandise des femmes... il y avait cela. Pour moi, c'était important qu'on puisse en parler et qu'on voie cela (Serge).

Virginie, quant à elle, s'est longuement imprégnée de photos, d'articles de presse. Elle se dit proche de Zina, la femme tchétchène dont nous suivons la trace jusqu'en Angleterre : une femme musulmane, mais aussi d'Occident, qui éprouve la guerre dans un espace qui pourrait nous être familier. Pour elle, plus que le maquillage, c'est le recours à la langue étrangère qui ouvre l'imaginaire : 
Les personnages étrangers sont plus faciles, parce qu'on est beaucoup moins réaliste. On parlait des gromelos ${ }^{14}$ au départ, ou alors quelques bribes de persan, et tout de suite, on était projetés dans une dimension beaucoup plus théâtrale. La preuve en est qu'il y a eu beaucoup, beaucoup de travail sur les équipiers de Sangatte. On n'arrivait pas à trouver les équipiers. Et je crois que c'est parce qu'on parlait français et que ça devenait tellement quotidien, tellement anodin. On relisait avec Delphine les premières moutures des textes de l'infirmerie de Sangatte, et on en rit maintenant, parce que c'était : " tiens, va vider la poubelle et ramène-moi un café » et on ne sortait pas de ça, c'était la glu. Il n'y avait pas de transposition, la force de la transposition (Virginie).

Ces personnages, il ne s'agissait pas seulement de les faire vivre, mais de faire venir leur monde. Pour cela, il fallait «ne pas venir avec des sacs de mots » (Ariane, répétition du 4 novembre 2002), mais au contraire donner toute sa place aux voix, aux sons et aux lieux.

Ce spectacle est beaucoup parti des lieux : comment allait-on faire exister ces lieux, qui étaient déterminants pour raconter l'histoire? ... Nous avons un jeu qui est proche d'un certain réalisme. On ne devait pas composer, " faire des personnages 》, mais essayer de vivre ces gens-là. Ces lieux, puisqu'ils donnaient une forme très nette, nous dégageaient du réalisme (Delphine).

Comment transformer un plateau en planisphère ? Comment faire venir le monde ? Du début à la fin du spectacle, les personnages ne mettent pas les pieds sur le plateau. Ils sont poussés sur des chariots, qui peuvent être de véritables roulottes ou des sortes de grandes planches à roulettes. Ce sont d'autres comédiens qui les poussent; le travail du comédien debout et parlant est ainsi doublé par celui, muet mais essentiel, des «pousseurs », qui jouent un peu le rôle d'ombre ou de chœur ${ }^{15}$. « Des pousseurs, on doit sentir la délicatesse, la maestria, comme celle d'un chef machiniste avec une dolly. Ils ont en eux les mêmes vagues qu'un metteur en scène» (Ariane, répétition du 18 novembre 2002). Comme avec le cinéma, un art avec lequel le Théâtre du Soleil entretient un dialogue actif, il devenait ainsi possible de faire entrer une infinité de lieux. On comprend pourquoi il était essentiel que les comédiens participent tous à l'élaboration de leurs lieux, même si c'était Serge et Duccio qui coordonnaient les tâches les plus complexes :

Jean-Charles (à Serge et Duccio) : Moi, un jour, je vous ai demandé le Taj Mahal, parce que j'étais un réfugié qui partait d'Inde, et vous avez refusé de construire le Taj Mahal. Je n'oublierai jamais.

Duccio: Non, je ne pense pas qu'on ait refusé, il y a des choses qui ne se sont pas faites. On n'a jamais dit: "ça, ce n'est pas possible ». C'est tout de même extraordinaire, à trente-huit, on ne s'est jamais censurés. C'est vrai qu'il y avait

14 Charles-Henri Bradier donne une définition de ce terme, qui revient fréquemment dans les conversations de travail : « Comme toujours au Théâtre du Soleil, on est passés par un langage imaginaire qu'on appelle le gromelo. Pour ce spectacle, on avait une somme de gromelos du monde entier qui arrivaient sur le plateau dès que les comédiens voulaient se mettre à parler, parce que le français donnait une lourdeur, un réalisme absolu à ce qu'on était en train de faire. C'était comme marcher sur le plateau au lieu d'y être sur un chariot ».

15 Les pousseurs dans ce spectacle établissent un lien avec des spectacles antérieurs Les Atrides et Tambour sur la digue, où avait été menée une profonde réflexion tant sur le chœur et sur l'ombre que sur la manière de se déplacer sur la scène. 
des propositions qu'on faisait qui ne prenaient pas, et il y en avait qui prenaient. Après, il y en a beaucoup qui se sont écrasées plus tard, en route.

\section{Ces chariots vous forcent à entrer dans un monde et à apporter un monde}

Lorsqu'on a commencé à travailler sur le spectacle, le mot qu'Ariane avait donné c'était : le départ, le voyage. Donc, elle voulait un camion. Tout a commencé par un camion, et le camion, on ne l'a pas quitté. C'était la métaphore idéale pour parler de tous les voyages : rouler, partir, avancer... (Charles-Henri).

Ce dispositif ne répond pas seulement à des fins techniques, c'est une puissante métaphore de ces vies d'errants à la fois détachés et attachés, vissés au maigre espace qui leur est alloué, un espace qui n'est pas un sol où ils puissent poser le pied. Mais, démentant, cette vision un peu « sociologique » et misérabiliste, le chariot qui glisse sur le vaste espace du plateau est aussi le lieu de l'apparition : «... tu n'es pas censé être sur un chariot, tu es sur ton état, tu es sur ta passion ». Le chariot est un socle ; il confère une qualité épique à ces trajectoires : "C'est comme dans le Kathakali, où les jupes sont les nuages et les dieux sont au-dessus ». La scène prend une dimension cosmique, et Sangatte devient un archipel, une galaxie : "Les chariots individuels, il faut qu'ils rentrent aussi avec leur petite lune de la planète, leur petite comète... $\rangle^{16}$.

Le chariot, c'est aussi ce qui permet d'opérer une densification de l'espace et une stylisation des actions et des situations. Le parallèle s'impose à mes yeux avec l'analyse que propose Jean Douchet ${ }^{17}$ du film de John Ford, The Stagecoach :

La visibilité immédiate est la condition essentielle. Il faut qu'on comprenne tout de suite. Tout le film va être sur ce qu'on comprend tout de suite. D'où la possibilité de travailler dans la diligence, parce qu'à ce moment-là, vous avez [...] une boîte, et des rapports de force immédiats, qui n'ont pas besoin d'être développés en-dehors, qui sont tout de suite là. C'est une façon de travailler une histoire en éliminant ce qui est le propre de l'histoire, c'est-à-dire « qu'est-ce qui va se produire ? », pour nous raconter l'histoire comme « de toute éternité », c'est-à-dire, d'emblée dans sa légende.

Pour le spectateur, le choc de la rencontre avec les chariots n'est pas immédiat. Chacune des deux parties est en effet ouverte par une scène très spectaculaire, qui mobilise toute la troupe, et où les chariots, qui ne sont pas poussés mais portés par les comédiens les plus athlétiques, sont cachés par des soies figurant les flots. Il s'agit dans l'un et l'autre cas du passage périlleux d'une étendue d'eau, même si ce n'est pas le même passage ni la même eau. La première partie, Le Fleuve cruel, commence par la traversée d'une rivière de montagne en crue. Cette rivière est une frontière entre deux territoires d'Asie centrale, probablement la Kirghizie et le Kazakhstan. C'est aussi la frontière, celle qui sépare les familles et les ethnies, celle pour laquelle on meurt, le lieu des ruptures et des

16 Tous ces propos ont été tenus par Ariane Mnouchkine lors des répétitions de novembre 2002.

17 Je cite ici quelques phrases de l'Entretien avec Jean Douchet : « Quintessence du Western » (17 minutes) repris dans le DVD collector de The Stagecoach (La Chevauchée fantastique) 2003. 
séparations, où s'affrontent les déshérités. Elle est comme la Voie Lactée dans le mythe chinois du Bouvier et de la Fileuse, qui sépare à jamais le couple stellaire. Ou, comme le disait Ariane à propos d'une autre improvisation : "c'est le passage de la mer Rouge ". D'emblée nous sommes avertis du prix de sang dont se sont acquittés certains voyageurs avant d'accoster nos rives.

Ce premier passage nous situe dans l'épique, dans l'Odyssée, mais aussi dans le temps. Pas le temps du désespoir, mais un temps qui ouvre sur un avenir : " après le passage... », « après... la catastrophe ». C'est le début du long voyage. La deuxième partie, Origines et destins, ouvre sur une scène elle aussi très spectaculaire, directement inspirée de l'épisode du Tampa, le cargo norvégien qui recueillit un navire en perdition chargé de 433 réfugiés repoussés par l'Australie ${ }^{18}$ et qui achemina sa cargaison humaine jusqu'en Nouvelle-Zélande. Cette fois, ce n'est plus un fleuve, mais un océan qui sépare, et les voyageurs ont déjà connu les refus, les fuites et les espoirs trahis. Cet épisode nous plonge dans un autre temps, un autre présent : celui qui va se figer pour bon nombre d'entre eux, incarcérés dans des camps de fortune ou plutôt d'infortune in the middle of nowhere.

Après ces deux scènes inaugurales, qui sont peut-être un rêve partagé par la troupe tout entière, vient le moment des récits plus intimes et des lieux précis. On peut désormais détailler les chariots : l'infirmerie de Sangatte, la première à apparaître, la roulotte où Timour et Claudia organisent leurs trafics sordides, une maison iranienne, un cinéma en Afghanistan, un atelier en Angleterre, mais aussi des espaces plus ouverts : une place à Kaboul, une mine dans le Caucase, des cabines téléphoniques un peu partout dans le monde, les grilles qui barrent aux réfugiés l'accès à Eurotunnel, grilles qu'il faudra sans cesse forcer ou escalader ${ }^{19}$. Comme pour les récits, on peut parler d'une multiplicité de styles allant de la simple évocation : "il y avait juste un bout de grillage, mais il y avait le cadre » (Jean-Charles) à la recréation des maisons précises et poétiques qui nous emportent là-bas.

Dans un premier temps, l'inspiration pour créer ces espaces a été cherchée dans les miniatures persanes, comme le rappelle Shasha :

Aux tout premiers jours, on ne savait pas où on allait. Il n'y avait même pas les chariots. Il y avait un plateau vide. La seule chose dont Ariane nous a parlé, c'était des miniatures persanes. Il y avait une grande table avec des livres sur l'Afghanistan, des feuillets sur Sangatte, des livres sur l'Iran, et Ariane nous a dit : " je ne sais pas où ça va, mais je vous donne une indication : c'est le cadre, le dessin et le cadre ». On a commencé à travailler avec ça : le cadre. Dans les scènes de Sangatte, les cabines resserrent l'espace, le cadrent. Il y a des personnages qui entrent, qui sortent, qui sont accrochés à la cabine ou assis et débordent un peu.

18 Astrid Grant, comédienne australienne, relie explicitement le travail de mémoire accompli par certains Australiens sur le passé violent de leur pays et l'accueil des réfugiés : «Nos ancêtres sont venus dans des bateaux et ils ont tué les Aborigènes. Il faut rappeler cette histoire, mais ce n'est pas quelque chose qui reste dans la vie actuelle. Il y a un mouvement qui lutte pour aider les réfugiés, et aussi des gens qui luttent pour nettoyer un peu l'histoire des relations entre les Australiens blancs et les Aborigènes. Ce sont les mêmes ».

19 Comment ne pas penser à d'autres prises d'assaut, plus récentes et plus tragiques encore : celles des enclaves de Ceuta et de Melilla, portes de l'espace Shengen ? Et devant l'indigne renvoi des réfugiés dans le désert, comment ne pas évoquer l'une des formes choisies pour le génocide des Arméniens en 1915 ? 
Ces scènes sont cadrées comme une page, et à la fois, il y a un élément qui sort du cadre un petit peu.

Ces miniatures sont les pages du livre qui nous est donné à feuilleter. Spectacle oral, polyphonique, Le Dernier caravansérail utilise aussi pleinement les ressources de l'écrit : la métaphore épistolaire nous est rappelée à maintes reprises, et les textes s'impriment sur le rideau dans leur élégante calligraphie : « On pourrait [...] parler, par analogie avec le terme performer pour l'acteur d'une performance d'écriture ${ }^{20}$ où le spectateur est convié à assister à l'écriture sur scène, où l'écriture émerge devant lui ». Plus encore qu'à Et soudain des nuits d'éveil auxquels ils étaient destinés, ces propos d'Anne Neuschäfer ${ }^{21}$ (Neuschäfer 2004b : 2) s'appliquent au Dernier caravansérail, un spectacle dans lequel " écriture » est à prendre aussi bien dans le sens de la production littéraire que de l'inscription graphique.

La miniature persane est aussi un art du cadre. Comme le rappelle Shasha, la nécessité de cadrer s'est imposée d'emblée. "Le cadre, pour nous c'était quelque chose de fondamental. C'est l'âme qui ressort de ce cadrage, comme l'âme ressort du masque », souligne Duccio.

\begin{abstract}
L'entrée et la sortie des chariots, ce n'est qu'un fondu au noir, une entrée de champ, une sortie de champ. Tout fonctionne sur l'apparition : quand le personnage entre en scène, le chariot s'arrête, paf! le regard se fixe sur le personnage à l'intérieur. On commence à regarder à ce moment-là, quand on a cadré avec le chariot. C'est en cela que le cinéma est important, qu'il nous a donné des leçons pour faire apparaittre (Charles-Henri).
\end{abstract}

La référence au cinéma n'est pas seulement dans la manière d'amener une scène, de cadrer, de faire apparaître. Elle passe par un dialogue avec les auteurs : Rossellini (Stromboli et peut-être aussi au moins pour une scène, la belle adaptation qu'il a faite des Fioretti de François d'Assises), John Ford, Charles Laughton ${ }^{22}$ et, pourquoi pas ? Tex Avery. Ce spectacle entretient un dialogue actif avec le documentaire ${ }^{23}$, le cinéma iranien et le cinéma muet.

Le caravansérail a fait le plein de voyageurs : la Bible, Lewis Caroll, Shéhérazade et ses Mille et une nuits sont, avec Zeami, les acteurs des théâtres d'Asie ${ }^{24}$, Chaplin et Murnau, les convives du banquet homérique. Nous trouvons la trace du conte, du mythe et de l'épopée non seulement dans certaines séquences, mais aussi dans la manière dont

Les italiques sont de l'auteur.

Elle cite Patrice Pavis (1993 : 3).

La scène qui se déroule dans le cinéma afghan est une référence directe à La nuit du chasseur qui, par son climat onirique, sa familiarité avec le mythe et l'épopée, est peut-être ce qui se rapproche le plus de certaines scènes du Dernier caravansérail.

Le film de Thierry Michel Iran, sous le voile de l'apparence est explicitement cité, mais il y a tous les films et reportages qui sont vus et commentés par les comédiens. Ce dialogue est incessant : lors de notre dernier entretien, alors que le spectacle est terminé, Ariane s'enthousiasme pour A l'Ouest des rails, le remarquable documentaire de Wang Bing sur le désastre d'une certaine modernité chinoise.

La familiarité, la complicité d'Ariane Mnouchkine avec les théâtres de l'Inde et de l'Asie orientale est bien connue. C'est un thème trop riche et trop complexe pour pouvoir être abordé ici. De nombreux articles lui ont été consacrés et sont repris sur le site. 
Ariane présente ses personnages dans le synopsis, écho sans doute de la manière dont elle a dû raconter ses histoires aux comédiens :

Apparaît ici pour la première fois Yosco, le passeur serbe, la tête de pont, le patron. Il évolue dans ce qu'il considère comme son territoire de chasse, ses tranchées, sa terre de guerre et de domination. Voilà celui qui choisit, détermine, autorise ou interdit à chacun de ces candidats le passage vers ce qu'ils espèrent, la tranquillité. Voilà celui que l'on paie, que l'on craint, que l'on peut maudire aussi, voilà Charon (Séquence 7 «A l'assaut des voies »).

Comme le dit excellemment Anne Neuschäfer: «En passant par le cycle des Shakespeare $^{25}$ et celui des Atrides $^{26}$, le Théâtre du Soleil s'est doté de la dimension épique et cosmique afin de raconter l'histoire de notre temps»(2004:13). Pour apprécier l'influence de Shakespeare sur ce spectacle, il suffit de citer les termes en lesquels sont présentés les passeurs de Sangatte dans le synopsis : "un réseau d'alliances secrètes, une hiérarchie de rois et d'officiers qui a pris les rênes de cette société désespérée... ». Leurs âpres et sanglants conflits sont présentés comme «le complot des méchants 》 dans Richard II. Plus loin, ce sont les grilles d'Eurotunnel qui sont décrites comme : « le champ de bataille de Henri IV, le lieu de tous les débuts et de toutes les fins ».

\section{Le Soleil travaille depuis toujours sur la guerre}

Entrelacs de motifs littéraires et artistiques, Le Dernier caravansérail est aussi traversé par des mémoires plus rudes, celles d'autres catastrophes, qui ne sont pas explicitement nommées. Le Rwanda et la destruction des juifs d'Europe sont des cataclysmes qu'Ariane Mnouchkine dit ne pouvoir représenter :

La tragédie accepte le théâtre, le théâtre accepte la tragédie, mais il y a quelque chose d'inhumain que je ne saurais pas faire : un spectacle sur l'épuration ethnique au Rwanda, la chambre à gaz décrite par Grossman dans Vie et destin (répétition du 17 décembre 2002).

La référence à la déportation, à la Shoah, à la Résistance constitue cependant la basse continue de ses compositions, l'une des sources profondes de son engagement humain, politique et artistique. Dans ce spectacle se repère aussi la trace de ce qui a anéanti la conscience occidentale en ce milieu du $20^{\mathrm{e}}$ siècle, et le caravansérail devient la chambre d'échos de cris lointains comme proches : «A l'entrée de la Tzigane, réminiscence lointaine des camps de concentration, mais il n'y avait rien que ça, ces quelques molécules de mémoire qui sont en l'air encore... » (Ariane, le 18 décembre 2002). Ou, encore, à propos d'une scène nocturne dans la forêt, où il s'agit de traverser un territoire au plus vite : «Ça m'a fait penser à la frontière suisse pendant la guerre : la grimpette dans la montagne de vacances et de villégiature devenue montagne de terreur et de crainte pour les Juifs qui essayaient de traverser » (Ariane, le 8 janvier 2003).

25 Richard II, La nuit des rois et Henri IV, représentés entre 1981 et 1984.

26 Iphigénie à Aulis, Agamemnon, Les Choéphores et les Euménides. Représentées à la Cartoucherie entre 1990 et 1992. 
Pour Charles-Henri Bradier, c'est le choc du 11 septembre, ressenti à Tokyo lors de la tournée de Tambours sur la digue ${ }^{27}$ qui serait à l'origine du spectacle. Il s'agirait donc d'un spectacle dont l'inspiration est venue "après la catastrophe », ou du moins après cette catastrophe-là. Ariane récuse cette analyse : "Peut-être que le 11 septembre a été comme pour tout le monde, une espèce de sceau, de cachet sur l'époque, mais il y a des catastrophes, soit naturelles, soit écologiques, plus grandes que le 11 septembre. Sans nier du tout l'importance du 11 septembre, ni sa cruauté ». Le moment déclencheur aurait plutôt été l'état de colère et d'indignation dans lequel la plongeait à chaque fois la visite du camp de détention des réfugiés à Villawood en Australie.

Ces questions ont leur importance, mais l'origine de ce spectacle remonte bien plus loin et renvoie à l'histoire du Théâtre du Soleil, une troupe qui a une histoire et qui travaille dans et avec l'Histoire :

Le Soleil travaille depuis toujours sur la guerre, sur la guerre civile, sur les déchirements intérieurs. Et pourquoi on travaille là-dessus? Probablement parce que c'est ce qui cause le plus de perte. C'est un sujet assez constant : l'irréparable, la perte irréparable. Il y a des défaites qui peuvent être des victoires, alors qu'il y a certaines défaites qui sont suivies de victoires, mais qui ne réparent en rien certains types de défaites (Ariane).

Depuis Sihanouk ${ }^{28}$, évocation puissante du génocide cambodgien (une tragédie qui n'avait cependant rien d' « humain »), et sans doute bien avant, mais de manière moins frontale, le Théâtre du Soleil mène, en harmonie avec Hélène Cixous, une réflexion approfondie sur l'histoire et ses déchirements : l'Indiade ${ }^{29}$, qui traite de la partition de l'Inde, les Atrides et le tragique enchaînement de la violence frappant hommes et dieux, la guerre que mènent les hommes à la nature dans Tambours sur la digue. Et qui dit guerre dit perte, exil, décentrement, refuge, thèmes qui étaient déjà ceux du spectacle précédent, Et soudain des nuits d'éveil :

\section{Le rapport qu'on avait avec certains étrangers en situation irrégulière, et certaines histoires qu'on nous avait racontées de réfugiés a fait qu'Ariane s'est rendu compte que le matériau qu'elle pouvait sortir de ces témoignages était un véritable matériau d'épopée. Il y avait déjà beaucoup d'histoires de héros ou d'Ulysse contemporains contenues dans le peu de choses qu'on avait entendues (Charles-Henri).}

Si elle arpente le sol de l'Histoire, Ariane Mnouchkine n'adopte cependant nullement le point de vue de l'historienne ou de l'ethnographe. Pas de regard éloigné. « J'ai besoin que le théâtre me raconte des histoires et qu'il me les raconte comme lui seul peut les raconter : légendairement et cependant droit dans les yeux ». Ces propos d'Hélène Cixous (1987 : 253) pourraient être les siens. Après tout, le sous-titre du spectacle est Odyssées,

27 Représenté à la Cartoucherie en 1999. 
et Ariane pourrait s'apparenter au barde qui plante sa tente sur quelques arpents de steppe et livre à un public de connaisseurs un fragment de l'épopée de Gésar. Et cependant, le jugement de Georges Banu (2002) doit être nuancé, lorsqu'il affirme : "Mnouchkine s'est affirmée toujours attirée par l'Histoire, mais, finalement, son théâtre a basculé du côté de la légende avec tout ce que celle-ci comporte comme immémorial et onirique ». La légende a sa place dans Le Dernier caravansérail, mais l'entreprise qui mobilisa la troupe pendant deux ans et qui la mobilise encore est plus ambitieuse : « Je considère que le théâtre doit être et politique et historique et sacré et contemporain et mythologique. Ce sont seulement les proportions qui changent de spectacle en spectacle ${ }^{30} \gg$. Le monde qu'elle entend nous présenter est plus complexe que celui des bardes et des conteurs, même si ces derniers y ont leur place, et il impose des parcours plus acrobatiques. Ce sont les voyageurs, ou du moins certains d'entre eux, qui viennent du pays des bardes. C'est après que le spectateur pourra s'imaginer avoir voyagé dans l'univers du conte ou de l'épopée. Lors du spectacle, il est accroché par «le harpon du présent» (Ariane, répétition du 9 janvier 2003). A ce sujet, les rappels à l'ordre sont incessants : «Tous ces départs sont des batailles, sont des sillons. Pas de fable, pas de conte. On n'est pas dans l'échappatoire»; "Pour raconter le monde, il faut que le public comprenne à chaque instant à quel endroit de la surface de la terre il est» ; « Tu dois comprendre la différence entre quelque chose de joli, beau, même bien fichu et quelque chose qui laisse une griffe sur mon cour. Là, tu enrobes mon cœur d'un petit sirop "; "Comment faire pour que le théâtre soit non seulement à la hauteur d'une simple photo, mais dépasse la simple photo?»

La grande réussite de ce spectacle faussement simple, faussement transparent est de nous confronter à la fois au présent et à la multiplicité des temps, la plupart des scènes qui nous sont présentées pouvant apparaître à la fois comme se passant « ce matin » et comme des récits faits « aux petits-enfants» ou « au paradis $»^{31}$. Béatrice Picon-Vallin (2000) parle fort judicieusement de conjugaison des temps à propos du travail d'Ariane Mnouchkine $^{32}$ : «Depuis trois décennies, le Soleil cherche inlassablement la manière de parler du présent de nos vies dans un style qui tisse le direct et l'indirect. Tout est affaire de conjugaison, donc de temps, et de combinaison, donc d'espace ». Nous pouvons donc à la fois recevoir ce spectacle comme une sorte de patchwork de lieux, de temps et de gens, accrochés là où ça se passe par le harpon du présent, et comme une polyphonie de récits renvoyant à un passé proche ou lointain. En effet, ce qui nous est donné à voir et à entendre n'est pas seulement une image de la dispersion et de la fragmentation du monde,

30 Propos d'Ariane Mnouchkine recueillis par G. Zaslavskij, « Un travail joyeux », Nezavissimaja gazeta (Moscou, 24-2-1998), cité dans Picon-Vallin (2000).

31 Cette piste nous est notamment suggérée par le très beau rituel des salutations, où les réfugiés ont maintenant quitté leurs chariots et marchent les uns vers les autres sur le sol, se touchent et s'embrassent. Une scène qui ne peut se passer qu' " au paradis », et qui évoque la fin, elle aussi « paradisiaque » de La ville parjure.

32 La conjugaison des temps ne se repère pas seulement d'une scène à l'autre, mais aussi à l'intérieur des scènes, où se heurtent des temporalités différentes. Cela nous est signifié dès la première scène, où c'est le refus des voyageurs d'attendre que s'apaise la colère du fleuve qui déclenche le conflit : « Chacun lutte pour sa survie : les réfugiés veulent passer à tout prix, le passeur être payé, le nautonier respecter la colère du fleuve. C'est aussi une confrontation entre les époques, la frontière peut-être aussi le lieu d'un tel éclat d'anachronisme » (Synopsis de la première séquence). 
mais aussi et surtout la reconstruction de fragments de la mémoire après la catastrophe, après leur catastrophe, individuelle ou collective. C'est ce que souligne Shasha, qui donne ainsi une des clés du spectacle :

\begin{abstract}
J'ai toujours un peu en tête, quand on racontait l'histoire de nos multiples personnages, qu'eux aussi avaient dî̀ à un moment de leur vie rencontrer quelqu'un comme Ariane, à qui ils avaient raconté leur histoire. Tout ce qu'on voit dans le spectacle, c'est ce qui leur est resté en mémoire (Shasha).
\end{abstract}

\title{
Après Babel
}

Passée par le travail de l'apprentissage et de la traduction, la multiplicité des langues parlées dans ce caravansérail induit un sentiment de proximité qui peut être trompeur, car la situation continue à se dégrader. L'aggravation de la précarité et de la menace nous est signifiée dans la forme même du spectacle. Le feuilletage des temps se présente en effet de manière différente dans les deux parties ${ }^{33}$. Dans la première, que l'on pourrait appeler « la galaxie Sangatte », il existe un espace commun minimal où peuvent parfois se rassembler quelques uns de ces voyageurs pour écouter les récits de leurs compagnons. C'est aussi le lieu où peut se faire entendre la nostalgie d'un monde qui n'est pas que terreur, l'évocation d'une beauté perdue, d'une douceur dont on trouve la trace dans le récit de Nadereh et les scènes afghanes, pour mieux nous poigner le cœur. Mais bientôt de tels récits ne pourront plus se faire en raison de la trop grande hétérogénéité des points de vue. C'est ce qui apparaît déjà dans la scène de la libération de Kaboul, à la fin de la première partie. Plusieurs personnages traversent le plateau: des femmes, qui sont sommées de remettre la burqa, les vainqueurs du jour qui déjà s'affrontent, un barbier qui fête de manière obscène sa victoire en rasant le cadavre d'un taliban exposé sur la place, et surtout les anciens seigneurs qui rejoignent à leur tour le flot des exilés en gagnant Londres au plus vite, relançant la fiction vers ce qui sera une des lignes de force de la deuxième partie. On meurt beaucoup, dans cette deuxième partie, dès la scène inaugurale, et ces morts nous sont données à voir frontalement. La dispersion gagne, la communauté précaire de Sangatte n'existe plus, il n'en reste que la trace ${ }^{34}$ :

Je me rappelle, quand Sangatte a été démantelé, on était quatre ou cinq à être partis avec Ariane sur cette espèce de champ dévasté. Pendant un an, deux ans, on y était tous allés voir, recueillir des bruits, des voix, et puis il y avait un bulldozer qui était passé ... il y avait les champs, il y avait des petits morceaux de tissu par terre et il continuait à y avoir les bruits des câbles. On regardait et il y avait la trace (Shasha).

Les expériments, scansion tragique ou poétique, adresse directe au spectateur, disparaissent presque totalement. Entre les scènes, les comédiens courent sur le plateau dans la plus grande urgence. La froideur des scènes d'interrogatoire en Australie, qui se

33 Le hangar est démonté le 23 décembre 2002 et le centre est fermé le 30 décembre.

34 Il ne faut cependant pas exagérer la dichotomie entre les parties. Il existe encore dans Origines et destins des espaces pour le rêve et la légende, et en particulier les trois épisodes de l'histoire du contrebandier, qui est une sorte de pendant moderne du mythe d'Orphée. Pour Maurice Durozier, « [ce] triptyque raconte une histoire d'amour au milieu de ces différentes odyssées et donne un petit repère au public dans cette absence de chronologie, dans cet océan de détresse». 
concluront par l'expulsion du demandeur d'asile, nous tire vers le documentaire d'un présent où la fiction s'abolit (il est significatif que le demandeur d'asile n'apparaisse plus en chair et en os, mais par le truchement d'un écran vidéo). Les retours au pays de plusieurs de ces voyageurs ne prêtent plus au récit, mais au constat: l'annihilation de Claudia, la pétrification du couple si sympathique de ces Afghans plus âgés, qui se voient brutalement remettre à leur place par un kapo local dans un camp du Pakistan. Y a-t-il dans ces scènes quelque chose que l'on souhaite raconter à ses petits-enfants ? Sans le moindre discours parasite nous est ainsi présentée l'image de la dégradation de notre monde : «On ne voit pas que l'Australie, on voit le monde d'aujourd'hui déjà, mais demain aussi », dit Ariane Mnouchkine à propos de la scène de l'interrogatoire australien. L'actualité lui a bien sûr donné raison, et l'on peut gager que l'Angleterre ne sera plus la terre de promesse qu'elle a pu être pour les premiers voyageurs. Virginie Colemyn a ces paroles prémonitoires à propos de la dernière scène du spectacle, qui se passe en Angleterre, et où encore le précaire et candide bonheur d'un pique-nique est gâché par les intégristes :

Moi, ce que je sens, c'est que tout d'un coup, on a passé peut-être des années sur les routes à chercher une terre promise, la terre promise qui est l'Angleterre, à tout prix, à tout prix, à n'importe quel prix et on arrive en Angleterre et là, il y a un énorme nuage, énorme, terrible, et comme une gangrène, comme quelque chose qui est là encore, et qui est là et qui est comme une rage de dents, qui va encore, encore, dicter sa loi. C'est ça qui me terrifie, c'est de sentir que même là-bas, et surtout là-bas, il y a ce terreau. Et Parissa, elle dit : " mais où est-ce qu'il est le monde, où est-ce qu'elle est cette terre-là, où je peux enlever le voile, et le mettre si j'ai envie de le mettre? "(Virginie).

Les anthropologues modernes fréquentent peu les caravansérails. Peut-être parce qu'il n'y en a plus, - reste à voir ! - et la lecture de cet article fait apparaître plus de différences que de convergences entre ce qui est avant tout une démarche artistique et ce qui voudrait se présenter comme discours scientifique. Mais, là où l'intuition du départ qui m'a fait frapper à la porte de la Cartoucherie garde à mes yeux tout son sens, c'est dans la recherche longue, soigneuse, obstinée du ton juste pour parler de l'autre sans trop trahir. L'anthropologue, pour autant qu'il ne s'égare pas dans une démarche de laboratoire, travaille sur les récits des autres et dans la langue des autres. Son outil est le langage et il est confronté à la nécessité de donner forme au matériau collecté pour construire un objet manipulable pour l'esprit. Ces préoccupations, cette responsabilité sont celles d'Ariane et de ses comédiens. Elles sont aussi celles du psychanalyste pour autant qu'il dépasse la « vignette clinique » pour tenter de construire au plus près un récit à partir du foisonnement d'éclats de vies souvent racontés dans les larmes. Produire un discours vrai, dans son incroyable ambition et sa possible ringardise, ce programme est celui de l'artiste, du psychanalyste et de l'anthropologue. Il passe par le souci de la forme bien plus que par les prises de pouvoir théorique ou les déclarations éthiques et politiques pompeusement assénées. Sur ce plan, la tribu frondeuse et voyageuse qui a planté sa tente depuis maintenant quarante ans dans le Bois de Vincennes nous montre la voie.

\section{Post scriptum écrit début 2006}

Depuis la fin de cet article, le Théâtre du Soleil est parti en tournée à New York (en juillet 2005) et à Melbourne (en octobre 2005). En janvier 2006, des comédiens iraniens 
du théâtre Shiah Bâzi de Téhéran sont venus jouer pendant un mois dans le théâtre qui leur avait été prêté à cette occasion.

En juin 2005, la troupe est partie pratiquement au grand complet à Kaboul pour organiser des stages de théâtre destinés à de jeunes comédiens afghans. Ceux-ci ont été hébergés pendant deux mois au Théâtre du Soleil pour y poursuivre leur formation; ils y sont restés deux mois et ils ont assisté à leur descente de l'avion à la dernière du spectacle qui a eu lieu le 5 février 2006. Certains d'entre eux, avec l'aide de deux comédiens du Soleil (Maurice Durozier et Shaghayegh Behesti) avaient monté Roméo et Juliette en Afghanistan. A Paris, ils ont travaillé sur Tartuffe!

Ce travail de formation de jeunes comédiens, une des activités essentielles du Théâtre du Soleil, s'est poursuivi de pair avec les répétitions d'un nouveau spectacle, dont le thème n'a pas encore été rendu public. 


\section{Références bibliographiques}

AвBas, Magnum, 2002. Iran Diary 1971-2002. Paris, Editions Autrement.

BANU, Georges, 2002. "Ariane Mnouchkine, la confiance faite au théâtre", dans Idem, Exercices d'accompagnement, d'Antoine Vitez à Sarah Bernhardt, pp. 58-70. Paris, Editions l'Entretemps.

BOULAT, Alexandra, 2002. Eclats de guerre. Paris, Les Syrtes images.

Cixous, Hélène, 1987. « Le lieu du Crime, le lieu du Pardon », dans Idem, L'Indiade ou l'Inde de leurs rêves, et quelques écrits sur le théâtre, pp. 253-259. Paris, Théâtre du Soleil.

Cixous, Hélène, «Un moment de conversion », fiche programme du spectacle Et soudain des nuits d'éveil. http://www.lebacausoleil.com.

Cramesnil, Joël 2004. La Cartoucherie, une aventure théâtrale. Paris, Editions de l'Amandier.

Derrida, Jacques, 1997. De l'hospitalité, Anne Dufourmantelle invite à répondre Jacques Derrida. Paris, Calman-Lévy.

Dusigne, Jean-François, 2003. Le Théâtre du Soleil. Des traditions orientales à la modernité occidentale. Paris, Centre national de documentation pédagogique.

FÉral, Josette, 2001 (1 $1^{\text {re }}$ édition, 1995). Trajectoires du Soleil. Autour d'Ariane Mnouchkine. Paris, Editions théâtrales.

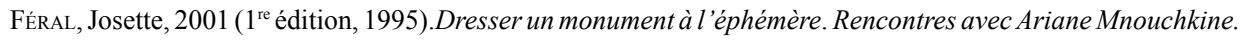
Paris, Editions théâtrales.

Grossman, Vassili, 2005. Vie et destin, Paris, Le livre de poche.

KRISTEVA, Julia, 1988. Etrangers à nous-mêmes. Paris, Fayard.

LemETRE, Jean-Jacques 1998. « Lire le corps avec la musique », in FÉRAL Josette (dir.), Trajectoires du Théâtre du Soleil, , pp. 41-71. Paris, Editions théâtrales.

Mnouchkine, Ariane, 2005. L'art du présent. Entretiens avec Fabienne Pascaud. Paris, Plon.

NeuschäFER, Anne,

2004. «1970-1975 : Ecrire une Comédie de notre temps - la filiation avec Jacques Copeau », texte écrit pour le site http://www.lebacausoleil.com (6 p.).

2004. «1975-1999 : De la création collective à l'écriture en commun », texte écrit pour le site http:// www.lebacausoleil.com (16 p.).

Pavis, Patrice, 1993. « Vers une théorie du jeu de l'acteur », Degrés n 75-76, pp. i1-i17.

Peschanski, Denis, 2002. « Autour de Sangatte », in Salmon Jacqueline, Sangatte. Le hangar. Calais, Galerie de l'ancienne poste et Musée de la Résistance et de la Déportation de l'Isère, Trans Photographic Press.

Picon-VAllin, Béatrice,

1995. «Une œuvre d'art commune (rencontre avec le Théâtre du Soleil), Théâtre/Public 124-125, pp. 74-83.

2000. «A la recherche du théâtre - le Soleil, de Et soudain des nuits d'éveil à Tambours sur la digue Les longs cheminements de la troupe du Soleil, Théâtre/Public 152, pp. 5-13.

2004. « Rêver à un espace qui permettrait toutes les apparitions (rencontre avec Guy-Claude François et Ariane Mnouchkine) », http://www.lebacausoleil.com.

SAlmon, Jacqueline (éd.), 2002. Sangatte. Le hangar, Calais : Galerie de l'ancienne poste et Musée de la Résistance et de la Déportation de l'Isère : Trans Photographic Press. 


\section{Les films cités dans l'article}

ForD, John, 1939. Stagecoach (La chevauchée fantastique), United Artists. DVD Ariès, Editions Montparnasse (DVD collector) 2003.

Laughton, Charles, 1955. The Night of the Hunter (La nuit du chasseur), Metro-Goldwin-Myer Pictures Inc. DVD, MGM Home Entertainment, 2004.

MICHEL, Thierry, 2002. Iran. Sous le voile des apparences. Les films de la Passerelle. DVD, Editions Montparnasse, 2004.

Rossellini, Roberto,

1949. Stromboli, Terra di Dio (Stromboli). RKO. DVD, Films sans frontières, s.d.

1950. Francesco, giullare di Dio (Les Fioretti de François d'Assises), Rizzoli Film. DVD, Criterion, 2005.

WANG Bing, Tiexi qu (A l'ouest des rails), 2003. Wang Bing Film Workshop. DVD MK2 éditions, 2004. 